\title{
Conductas de riesgo psicosocial en alumnos del Plantel 01 (Colegio de Bachilleres de Chiapas)
}

\section{Behavior of psychosocial risk in students of the Plantel 01 (Colegio de Bachilleres de Chiapas)}

HERNÁNDEZ-GORDILLO, José Luis†, ESCALANTE-GORDILLO, Aurora Fernanda, LOPEZHERNÁNDEZ, Luvia Citlalli y RUÍZ-FLORES, Ana María

Universidad de Ciencias y Artes de Chiapas. Libramiento norte poniente 1150. Col. Caleras Maciel. C.P 29039. Tuxtla Gutiérrez, Chiapas

ID 1 ${ }^{\mathrm{er}}$ Autor: José, Hernández Gordillo / CVU CONACYT ID: 244814

ID $1^{\text {er }}$ Coautor: Aurora Fernanda, Escalante-Gordillo

ID $2^{\mathrm{do}}$ Coautor: Luvia Citlalli, López-Hernández

ID $3^{\text {er }}$ Coautor: Ana María, Ruiz-Flores

DOI: $10.35429 / J P D .2019 .10 .3 .8 .19$

Recibido 01 de Octubre, 2019; Aceptado 29 de Diciembre, 2019

\begin{abstract}
Resumen
La presente investigación cuantitativa aborda a nivel descriptivo comparativo el tema de factores de riesgo psicosocial en el nivel de bachillerato del Plantel 01 "Tuxtla Terán" del Colegio de Bachilleres de Chiapas (COBACH) ubicado en la ciudad de Tuxtla Gutiérrez; realizando una comparativa entre grupos de alumnos regulares con alumnos irregulares. La intervención es producto del trabajo interinstitucional del cuerpo académico "educación y procesos sociales contemporáneos" de la Universidad de Ciencias y Artes de Chiapas (UNICACH) y de la Fiscalía de Derechos Humanos (Dirección de Atención a Víctimas) adscrita a la Fiscalía General del Estado de Chiapas. Tiende a pensarse que los alumnos repetidores son proclives a conductas de riesgo y a decir de las autoridades educativas, ser objetos de bajo rendimiento académico, reprobación o deserción escolar. Las conductas de riesgo psicosocial suelen verse acrecentadas en la adolescencia, siendo la edad un factor de manera indirecta para realizar conductas transgresivas o delictivas que pueden poner en riesgo su salud física y mental. Por ejemplo, en el uso y abuso de sustancias tóxicas como drogas, alcohol, intento de suicidio, conducta antisocial, conducta sexual de riesgo o embarazo adolescente.
\end{abstract}

\begin{abstract}
The present quantitative research addresses, at a comparative descriptive level, the issue of psychosocial risk factors at the high school level of school center 01 "Tuxtla Terán" of Colegio de Bachilleres de Chiapas (COBACH) located in the city of Tuxtla Gutiérrez; making a comparison between groups of regular students with irregular students. The intervention is a product of the inter-institutional work of the academic body "education and contemporary social processes" of the Universidad de Ciencias y Artes de Chiapas (UNICACH) and of the Procurator's Office of Human Rights (Directorate of Attention to Victims) attached to the General Procurator's Office from Chiapas. It tends to be thought that repetitive students are predisposed toto risky behaviors and, in the words of the educational authorities, to be objects of low academic performance, school failure or dropout. Psychosocial risk behaviors tend to be increased in adolescence, with age being an indirect factor in transgressive or criminal behavior that may put their physical and mental health at risk. For example, in the use and abuse of toxic substances such as drugs, alcohol, suicide attempt, antisocial behavior, risky sexual behavior or teenage pregnancy.
\end{abstract} Bachilleres de Chiapas). Revista de Didáctica Práctica. 2019. 3-10: 8-19.

\footnotetext{
$\dagger$ Investigador contribuyendo como primer autor.
} 


\section{Introducción}

El desarrollo del adolescente implica que este se desenvuelva en condiciones óptimas en el contexto familiar y social. Pero muchas veces al carecer de esto y aunado a que la adolescencia es una etapa de cambios biológicos y psicológicos, conllevan a enfrentarlo a dificultades en su ciclo vital pudiendo esto crear un ambiente proclive a incidir en conductas de riesgo psicosocial.

Se aborda una comparativa entre grupos únicos y regulares del Colegio de Bachilleres de Chiapas (COBACH) Plantel 01. Los primeros son recursadores sea por bajo rendimiento escolar, mala conducta o con problemas de ámbito externo al educativo son etiquetados por la misma escuela como alumnos problema, se cree entonces que esto es un factor para que incidan en conductas de riesgo psicosocial, dando por hecho que los grupos regulares no son vulnerables a incurrir en estas conductas.

Mencionan Valenzuela M., Ibarra A. y Zubarew T. (2013) las conductas de riesgo "implican un deterioro considerable en la salud del adolescente afectando su calidad de vida, la inserción con igualdad de oportunidades en la sociedad. Por esta razón los grupos irregulares se han vuelto foco de atención para los directivos puesto que al creer que son grupos problema ya no se puede trabajar de la misma que con otros grupos. De tal forma que se decidió realizar diversos test a grupos regulares y grupos únicos con la intención de conocer si este puede ser un factor que ponga a los grupos irregulares como vulnerables y poder entonces focalizar una mejor atención para poder incidir de manera positiva a su desarrollo.

\section{Justificación}

Las conductas de riesgo psicosocial en los adolescentes se ha convertido en un problema que no solo afecta el ámbito educativo si no el desarrollo global del adolescente ya sea en un embarazo no deseado, abuso de sustancias como alcohol y drogas, infecciones de transmisión sexual, depresión que derive en intentos de suicidio, suicidio o muerte temprana. Ya que cuando el adolescente incide en estas conductas pone en riesgo tanto su estado emocional, como su salud física y la de otros, y no solo de forma temporal si no también con efectos a largo plazo.
Por tanto, es importante conocer si el estado de grupo irregular con todo lo que conlleva esto es un factor para que los adolescentes incidan en conductas de riesgo o realmente no es algo inherente. Ya que en la actualidad a pesar de que hay mucha información acerca de los métodos anticonceptivos, las infecciones de transmisión sexual y las consecuencias de utilizar drogas o alcohol, parecería que no es suficiente para controlar este fenómeno, ya que por más información y campañas de prevención que se les dé a los adolescentes en la escuela el resultado parecería ser el contrario.

\section{Problema}

Lo mencionado con anterioridad nos induce a considerar que la problemática principal a desarrollar en el artículo es la incidencia de conductas de riesgo psicosocial del Plantel 01 "Tuxtla Terán" de los grupos únicos y regulares, para comparar la incidencia de casos de consumo de sustancias, depresión e ideación suicida de conductas sexuales de riesgo y relación con padres. En ese sentido se pretende trabajar con la interrogante ¿La condición de grupo escolar único es factor para incidir en conductas de riesgo en el bachiller del Plantel 01 “Tuxtla Terán”?

\section{Hipótesis}

Los alumnos de los grupos únicos del Plantel 01 del Colegio de Bachilleres de Chiapas son más proclives que los alumnos del grupo regular a incidir en conductas de riesgo psicosocial.

\section{Objetivos}

\section{Objetivo general}

Identificar la incidencia de conductas de riesgo psicosocial de los alumnos grupo único y regular

\section{Objetivos específicos}

Comparar la incidencia de casos de consumo de sustancias, depresión e ideación suicida de conductas sexuales de riesgo y relación con padres en bachilleres de los grupos únicos y regulares. 


\section{Marco teórico}

La adolescencia ofrece oportunidades para el crecimiento, no sólo en las dimensiones físicas sino también en competencia cognitiva y social, autonomía, autoestima, e intimidad. Este periodo también conlleva riesgos, algunos problemas para manejar todos estos cambios a la vez y es posible que necesiten ayuda para superar los riesgos que encuentran a lo largo del camino para llegar a la adultez (Papalia, 2009).

La adolescencia constituye un periodo evolutivo caracterizado por numerosos cambios biológicos, psicológicos y sociales, siendo habitual en esta etapa los conflictos familiares entre progenitores e hijos/as. Los hijos e hijas reclaman sobre todo autonomía y una relación más igualitaria (Rodrigo, García, Márquez y Triana, 2005), y esto crea situaciones de conflicto familiar con más frecuencia que en las etapas evolutivas anteriores. En estas situaciones puede haber tanto agresión verbal como física de hijas o hijos hacia las madres y padres de diferentes niveles de severidad.

La familia sigue siendo el más importante agente de socialización, a pesar de todos los cambios experimentados en las últimas décadas en cuanto a su estructura o el cambio en los roles de los padres y madres. La comunidad científica se ha interesado por el estudio de las posibles repercusiones que dicha transformación puede acarrear para el desarrollo psicológico infantil (Antolín, Oliva y Arranz, 2009). Los cambios en la estructura familiar se han asociado tradicionalmente a la presencia de problemas de conducta y/o conductas delictivas así como a problemas en las relaciones paternofiliales (Hetherington y Henderson, 1997).

El clima familiar positivo hace referencia a las prácticas de socialización de la familia que fomentan la confianza, la autonomía, la iniciativa y los lazos afectivos para el desarrollo de hijas e hijos estables y competentes (Werner, 1989).

Hay evidencia empírica de que el clima familiar positivo es un factor protector de la violencia filio-parental (VFP) evaluado a través de la cohesión familiar, relaciones paternofiliales positivas y conductas prosociales.
Las relaciones positivas entre padres/madres e hijos/as se han asociado con un menor riesgo de abuso verbal o físico de las y los adolescentes hacia sus madres y/o padres (Jaureguizar e Ibabe, 2012; Pagani et al., 2003) y con un mayor desarrollo de conductas prosociales en el ámbito familiar (Jaureguizar e Ibabe, 2012; Romano, Tremblay, Boulerice y Swisher, 2005).

Además, en este estudio se encontró una relación inversa entre las conductas violentas y conductas prosociales de las hijas e hijos hacia los progenitores. Parece que las relaciones padres-hijos, basadas en el afecto y la comunicación, sirven para prevenir la violencia filio-parental y las conductas antisociales en general.

Por el contrario, el clima familiar negativo es uno de los factores de riesgo más conocidos de la violencia filio-parental y está caracterizado por un elevado nivel de conflicto familiar, bajo nivel de cohesión y presencia de violencia familiar (violencia marital y violencia de padres a hijos).

Los adolescentes agresivos describen a sus progenitores como personas que les rechazan, son sobre protectoras y/o menos cálidas emocionalmente (Aluja, Del Barrio y García, 2005). También se ha comprobado empíricamente que el conflicto familiar es un predictor tanto de las conductas violentas hacia las figuras de autoridad como de las conductas antisociales (Jaureguizar e Ibabe, 2012).

Por tanto, como se menciona anteriormente un clima familiar negativo aunado a los cambios físicos y psicológicos que conlleva la adolescencia puede volverse un factor de riesgo.

Los factores de riesgo son aquellos elementos que tienen una gran posibilidad de originar o asociarse al desencadenamiento de un hecho indeseable, $\mathrm{o}$ de una mayor posibilidad de enfermar o morir, pueden ser la causa de un daño o actuar como moduladores del mismo si influyen en él, un ejemplo claro podría ser el alcohol y la conducción. (Orbegoso, B. 2014) Según Gómez Carda (1999, p. 4): 
Denomina factores de riesgo a circunstancias o eventos de naturaleza biológica, psicológica o social que favorecen la posibilidad de que se produzca unos problemas. $\mathrm{Su}$ conocimiento permite establecer prioridades de atención del problema de acuerdo con la forma como se relaciona dichas circunstancias o eventos y las características del propio adolescente. Por ende, al existir factores de riesgo en la vida del adolescente este tiene una mayor probabilidad de incidir en conductas de riesgo durante la etapa de la adolescencia. La teoría de conducta problema de Jessor, nos ofrece subsidios más específicos para comprender el comportamiento del desarrollo adolescente. Este modelo abarca elementos (o sistemas) tales como cognición, aprendizajes, relaciones interpersonales y constructos intrapersonales en sus relaciones sociopsicológicas, buscando explicar por qué los adolescentes presentan determinados comportamientos de riesgo.

\section{Éstos engloban conductas que} representan riesgo para la salud personal, conductas relacionadas a la delincuencia y de desadaptación escolar. Concretamente, Jessor (1992, pág. 374) define como "las conductas problema como aquel tipo de actividades que son socialmente definidas como problemáticas, que son fuente de preocupación o que se consideran indeseables por las normas convencionales". Además, su ocurrencia ilícita algún tipo de respuesta de control; esta respuesta puede ser leve (la reprobación, la amonestación por parte de los adultos) o puede ser más severa (por ejemplo, el encarcelamiento). De acuerdo con esta teoría, la conducta problema es propositiva, instrumental, funcional. Los modelos enfocados en el riesgo, han demostrado ser especialmente útiles en el trabajo con los jóvenes, Dryfoos (1990) conceptualiza este modelo describiendo factores antecedentes a las conductas de riesgo, los que están ligados a características socio demográficas (sexo, edad, etnicidad, educación de los padres, extracción rural o urbana), factores personales (nivel educacional, notas de la escuela, religiosidad, personalidad y sus características, etc.), familiares (grado de cohesión, flexibilidad, calidad de , la comunicación, etc.), comunitarios y (características del sistema escolar, de pares, de los medios de comunicación) del contexto sociocultural amplio ( situación de empleo, oportunidades educacionales, nivel de ingresos, etc.).

ECORFAN $^{(\mathbb{R}}$ Todos los derechos reservados
Por tanto, la conducta de riesgo la define como "La mayor probabilidad de que se produzcan consecuencias negativas para la salud" (Dryfoss 1990, Pág. 280). Estas constituyen un conjunto amplio, como por ejemplo farmacodependencia, conducta sexual temprana, delincuencia, conductas violentas, deserción escolar, bulimia y anorexia, y problemas específicos de la psicopatología como la depresión adolescente. Las cuales a continuación se abarcarán brevemente.

Los problemas escolares que muchas veces se manifiestan en inasistencias, la falta de interés en las clases, la poca motivación o concentración deficiente, así como la baja energía mental, son señales que advierten síntomas de depresión en adolescentes.

Existen quienes se autoinfligen con cortaduras o a través de la alimentación, cayendo en trastornos como la anorexia o la bulimia, síntomas inequívocos de que están sufriendo depresión y la muestran con actos que obligan a la atención inmediata.

La Organización Mundial de la Salud (OMS) define a la depresión como un trastorno mental frecuente, que se caracteriza por la presencia de tristeza, pérdida de interés o placer, sentimientos de culpa o falta de autoestima, trastornos del sueño o del apetito, sensación de cansancio y falta de concentración. Esto puede llevar al adolescente a otra conducta de riesgo, como lo es la ideación suicida. Henri E. (2008), sostiene:

"La idea de suicidio puede germinar en condiciones psíquicas variadas: generalmente son los 'trastornos afectivos' de tono depresivo y ansioso los que la engendran; otras veces se debe a trastornos de obnubilación de la conciencia con alteraciones de la actividad intelectual y ansiedad paroxística; por último puede estar ligada a todo un conjunto de ideas y de creencias delirantes en relación a las cuales constituye una 'reacción', tanto es verdad que el suicidio, sea normal o patológico, expresa una intención fundamental, es decir una pulsión, una fuerza de organización y de liberación que emana de las fuentes más primitivas del instinto, de lo que Freud llamó pulsión de muerte". 
Otra de las conductas de riesgo más frecuentes en los adolescentes en la actualidad es el consumo de sustancias nocivas para su salud. El uso de drogas durante la infancia y adolescencia provoca alteraciones físicas, psicológicas y sociales. Además de su asociación con accidentes de tráfico, homicidios y suicidios, también se relaciona con actividad sexual temprana, fracaso escolar y delincuencia, además de las posibles consecuencias en la edad adulta, como las enfermedades cardiovasculares, alteraciones mentales etc.

De acuerdo a la Encuesta Nacional de Adicciones (2016) el índice de adolescentes que presento dependencia de alcohol incremento significativamente con la medición del 2002, pasando de $2.1 \%$ a $4.1 \%$ en 2011 . Y con respecto a la edad de inicio de consumo de alcohol, en el estudio del 2008 el $49.1 \%$ de las personas mencionó haber consumido por primera vez a los 17 años o menos, mientras que en el 2011 ese porcentaje se incrementó a 55.2\%. La edad de inicio de 16.6 años en hombres, y en las mujeres se encontró alrededor de los 19 años en 2011.

Así como también caer en conductas sexuales de riesgo en las cuales los adolescentes tienden a iniciar relaciones sexuales completas cada vez más precozmente, el uso inconstante del preservativo y la no utilización de otros métodos anticonceptivos. También se observan importantes déficits de información sobre sexualidad entre los jóvenes

La mayoría de las personas inician su vida sexual durante la adolescencia. Pero el uso de anticonceptivos y preservativos es limitado entre los jóvenes. Cada año, el $11 \%$ de los alumbramientos producidos en el mundo (unos 16 millones) corresponde a niñas de entre 15 a 19 años. El número de abortos en adolescentes supera los 2,5 millones (OMS, 2017).

\section{Metodología de la investigación}

La presente investigación de enfoque cuantitativo, se basa en un estudio descriptivocomparativo, aplicado a una población de 284 alumnos adscritos al Plantel 01"Tuxtla Terán" del Colegio de Bachilleres de Chiapas.

\section{Tipo de investigación}

En la realización del estudio descriptivo el investigador sabe lo que quiere investigar en cuanto a objetivos y diseño, pretendiendo describir algunas características fundamentales de conjuntos homogéneos de fenómenos, tal como se presentan en la realidad, utilizando criterios sistemáticos que permiten poner de manifiesto su estructura y comportamiento, sin llegar a explicar la naturaleza de las variables en cuestión o de la interacción existente sobre esta. (Mario Tamayo y Tamayo, 1987).

Así como también se utilizará un estudio comparativo, que para Sartori (1984) el método tiene como objetivo la búsqueda de similitudes y disimilitudes. Dado que la comparación se basa en el criterio de homogeneidad; siendo la identidad de clase el elemento que legitima la comparación, se compara entonces lo que pertenece al mismo género o especie. Las disimilitudes se presentan como lo que diferencia a la especie de su género, y esto no es lo mismo que señalar las variaciones internas de una misma clase; por lo cual se requiere de un trabajo sistemático y riguroso que implique la definición previa de las propiedades y los atributos posibles de ser comparados.

\section{Métodos teóricos}

Encuesta “(...) es una búsqueda sistemática de información en la que el investigador pregunta a los informantes sobre los datos que se desea obtener" (Díaz, 2001, p.13).Para determinar las conductas de riesgo que predominan en esta población, se aplicó:

Prueba de detección de consumo de alcohol, tabaco y sustancias (ASSIST, por sus siglas en inglés) la cual fue desarrollada para la Organización Mundial de la Salud (OMS) por un grupo internacional de investigadores y médicos como una herramienta técnica para ayudar a la identificación temprana de riesgos para la salud y trastornos debido al uso de sustancias en la atención primaria de salud, la atención médica general y otros entornos. 


\section{Promedio}

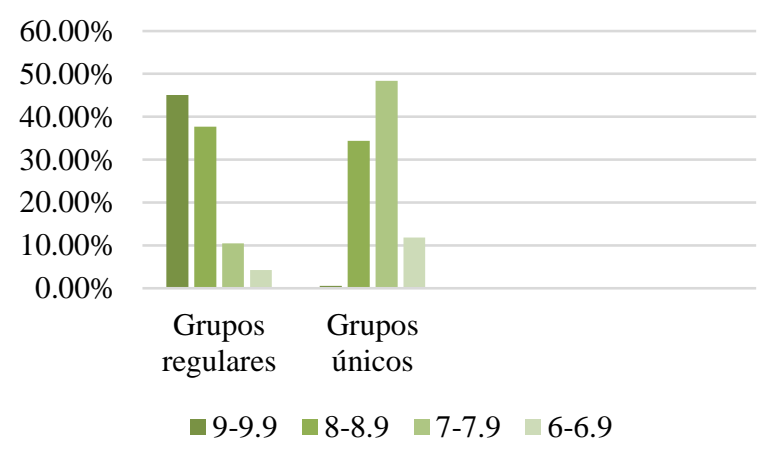

Gráfico 1

Así como también un cuestionario de factor de riesgo psicosocial y salud ocupacional el cual consta de 7 escalas dentro de las cuales incluye ideación suicida, depresión, impulsividad, autoestima, sexualidad y relación con padres. Estas técnicas serán utilizadas con la finalidad de tener un panorama de las incidencias en conductas de riesgo psicosocial que hay en los alumnos, así como a que conducta son más propensos a incidir cada grupo.

\section{Resultados}

A partir de la aplicación de los instrumentos Psicosocial de adolescentes y la prueba de detección de consumo de alcohol, tabaco y sustancias (Alcohol, Smoking and Substance Involvement Screening Test, ASSIST ASSIST) se determinaron conductas de riesgo organizadas en unidades de análisis de sexualidad, depresión, ideación suicida, relación con papá y relación con mamá e incidencia en el consumo de sustancias. La población encuestada integrada por 284 bachilleres se dividió en grupos regulares y únicos con 162 y 122 respectivamente.

\section{Promedio}

En los grupos regulares encontramos que los alumnos que tiene un promedio que va del 9-9.9 ocupan el $45.1 \%$ de la población, los que tienen $8-8.9$ un $37.7 \%$, de $7-7.9$ un $10.5 \%$ y los que tienen de 6-6.9 un 4.3\%. Los alumnos de grupos únicos obtuvieron un promedio que va de 9-9.9 es el .6\%, los tienen 8-8-9 un 34.4\%, de 7-7.9 un 48.4, y los que tiene de 6-6.9 un $11.8 \%$, lo que nos indica que en los grupos regulares los alumnos tiene un promedio más alto que los alumnos de grupos únicos, que puede verse reflejado en su rendimiento académicos de ambos grupos.

\section{Sexualidad}

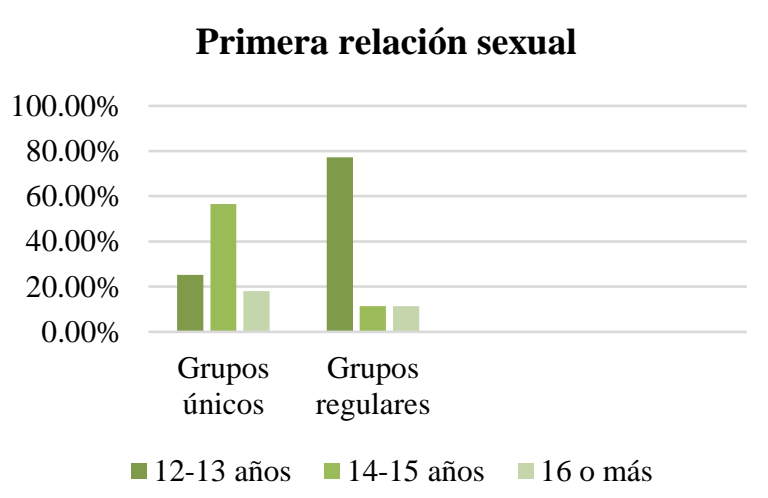

Gráfico 2

En cuanto a relaciones sexuales, los alumnos de grupos únicos manifiestan en mayor número haber tenido relaciones sexuales en los últimos meses, siendo el $65.6 \%$ de la población equivalente a 80 adolescentes que afirma que sí, a diferencia de los alumnos de grupos regulares siendo un $18.4 \%$ equivalente a 29 adolescentes que afirma que sí. Sumado a lo anterior podemos ver que la mayoría de los alumnos de grupos únicos se cuida al tener relaciones sexuales con un $60.5 \%$ equivalente a 46 adolescentes a diferencia de los alumnos de grupos regulares de los cuales el $27.80 \%$ con un equivalente a 10 adolescentes que utilizan condón en sus relaciones sexuales. Esto nos indica que hay un mayor número de adolescentes en los alumnos de grupos únicos que han tenido relaciones sexuales, aunque a pesar de esto podemos percatarnos que es mayor la población de alumnos de grupos regular los que no utilizan preservativos.

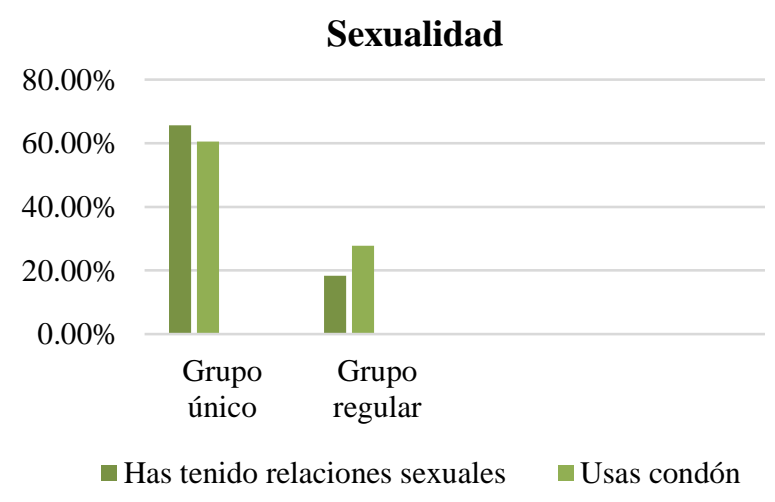

Gráfico 3

HERNÁNDEZ-GORDILLO, José Luis, ESCALANTE-GORDILLO, Aurora Fernanda, LOPEZ-HERNÁNDEZ, Luvia Citlalli y RUÍZFLORES, Ana María. Conductas de riesgo psicosocial en alumnos del Plantel 01 (Colegio de Bachilleres de Chiapas). Revista de Didáctica Práctica. 2019 


\section{Edad de la primera relación sexual}

Podemos ver que el $56.6 \%$ equivalente 47 alumnos de grupos únicos tuvo su primera experiencia sexual durante los 14-15 años, un $25.3 \%$ equivalente a 21 adolescentes tuvo su primera relación sexual a los 12-13 años y un $18.1 \%$ equivalente a 15 adolescentes a los 16 años o más, mientras los alumnos de grupos regulares un $77.3 \%$ con un equivalente a 34 adolescentes tuvo su primera experiencia de 1213 años de edad, un $11.4 \%$ equivalente a 5 alumnos de 16 o más años, y un $11.4 \%$ equivalente a 5 alumnos de 14-5 años de edad. Dentro de lo cual observamos que hay una población significativa entre los alumnos de grupos regulares que tuvo su primera relación sexual a una etapa muy temprana de su desarrollo, lo cual puede exponer a los adolescentes a una situación de riesgo.

\section{La escala de sintomatología depresiva (CES- D)}

Esta escala evalúa la sintomatología depresiva una semana previa a su contestación, se escogieron los reactivos más relevantes que pueden detectar esta condición en los alumnos, (Sentí que no podía quitarme la tristeza ni con la ayuda de mi familia o amigos, me sentí deprimido, me sentía solo, sentía que mi familia estaría mejor si estuviera muerto, pensé en matarme). El $16 \%$ equivalente a 26 alumnos de grupos regulares presentan tristeza de 5-7 días, mientras que dentro de los grupos únicos la población es de $6.6 \%$ equivalente a 8 adolescentes, de igual forma, el $15.8 \%$ equivalente a 25 alumnos de grupos regulares comenta que se sintió deprimida de 5-7 días a diferencia de los alumnos de grupos únicos dentro de los cuales un $4.9 \%$ equivalente a 6 personas comenta que se han sentido deprimida de 5-7 días. El 25.9\% equivalente a 41 alumnos de grupos regulares manifiestan que se ha sentido solo de 5-7 días por parte el $6.7 \%$ equivalente a 8 alumnos de los grupos únicos se han sentido solo de 5-7 días.

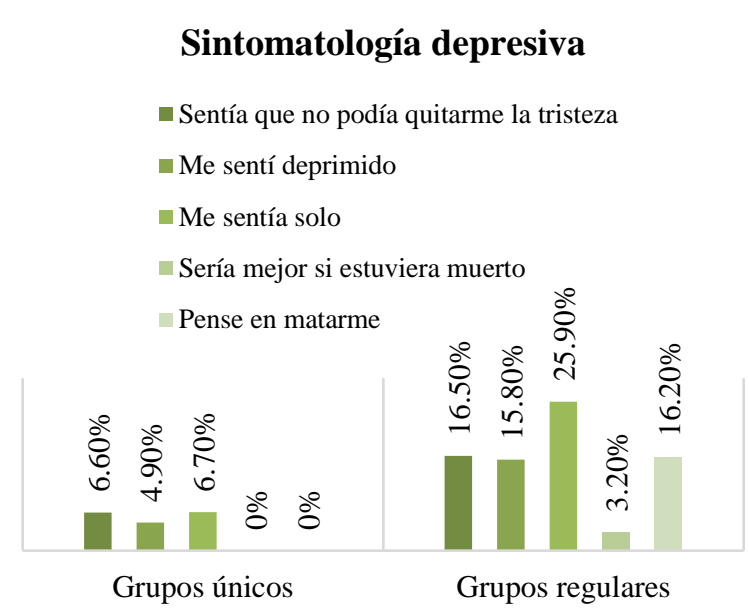

Gráfico 4

Por otra parte, podemos observar que el $3.25 \%$ equivalente a 5 alumnos de grupo regular comenta que ha pensado de 5-7 días de la semana anterior. que sería mejor si estuviera muerto. Así como también que un $16.2 \%$ equivalente a 25 alumnos de grupos regulares pensó en matarse de 5-7 días de la semana anterior a la aplicación de la encuesta. Mostrando una diferencia significativa entre los dos grupos indicándonos que hay una mayor incidencia en una sintomatología depresiva por parte de los alumnos de grupos regulares.

\section{Escala de relación con papá}

Los siguientes gráficos muestran los reactivos más importantes para conocer como es la relación de los alumnos de grupos únicos y regulares con papá.

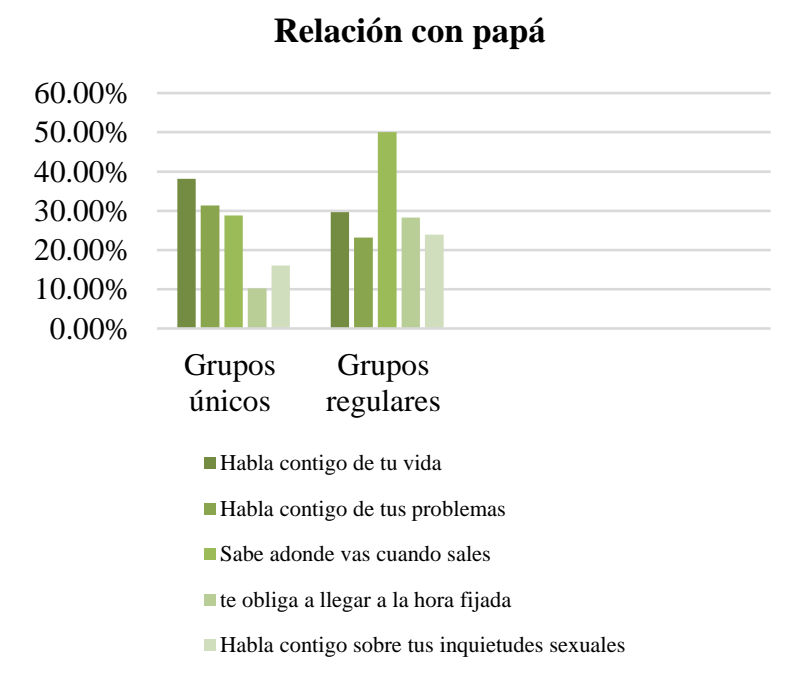

Gráfico 5 
En el Gráfico de los alumnos únicos podemos notar que un $38.1 \%$ equivalente a 45 adolescentes habla con sus papás de su vida con mucha frecuencia, mientras que dentro los alumnos regulares hay un $29.7 \%$ equivalente a 41 adolescentes, de igual forma, el $31.1 \%$ equivalente a 37 alumnos de grupos únicos mencionan que sus padres hablan con ellos de sus problemas a diferencia de los alumnos de grupos regulares dentro de los cuales un $23.2 \%$ equivalente a 32 adolescentes comenta que sus padres hablan con ellos sobre sus problemas.

El 50\% equivalente a 69 alumnos de grupos regulares mencionan que sus padres saben adónde van cuando salen mientras que dentro de los alumnos de grupos únicos lo mencionan un $28.8 \%$ equivalente a 34 adolescentes, así como el $28.3 \%$ equivalente a 39 alumnos de grupos regulares nos comentan que su padre lo obliga a llegar a la hora fijada a diferencia de los alumnos de grupo único donde podemos notar que hay un $10.2 \%$ equivalente a 12 adolescentes.

Por otro lado, el 23.9 equivalente a 33 alumnos de grupo regular nos dice que habla con sus padres sobre sus inquietudes sexuales, a diferencia de los alumnos de grupos únicos dentro de los cuales el $16.1 \%$ equivalente a 19 adolescentes nos mencionan lo mismo.

Pudiendo observar con esto que no existe una diferencia significativa entre la relación que llevan los alumnos de grupos únicos y regulares con su padre en cuanto hablar con ellos sobre su vida y sus problemas, pero notamos también una diferencia significativa respecto a un mayor control por parte de su padre en cuanto a los lugares adónde sale, así como en la hora de llegada, así como una mejor comunicación respecto a inquietudes sexuales.

\section{Escala de relación con mamá}

Las siguientes graficas muestran los reactivos más importantes para conocer como es la relación de los alumnos de grupos únicos y regulares con mamá.

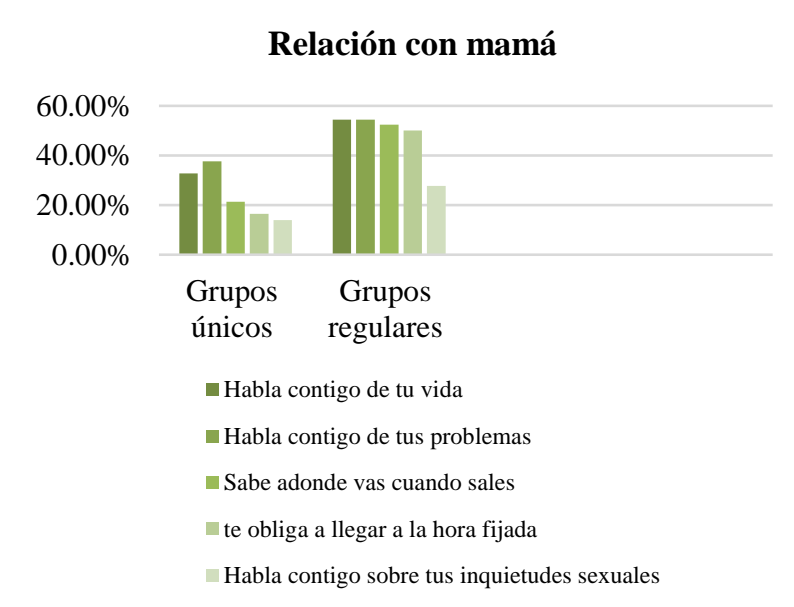

Gráfico 6

En el gráfico de los alumnos únicos podemos notar que un $32 \%$ equivalente a 40 adolescentes habla con sus papás de su vida con mucha frecuencia, mientras que dentro los alumnos regulares hay un $54.4 \%$ equivalente a 86 adolescentes, de igual forma, el $37.7 \%$ equivalente a 46 alumnos de grupos únicos mencionan que sus madres hablan con ellos de sus problemas a diferencia de los alumnos de grupos regulares dentro de los cuales un 54.4\% equivalente a 86 adolescentes comenta que sus madres hablan con ellos sobre sus problemas.

El $52.5 \%$ equivalente a 83 alumnos de grupos regulares mencionan que sus madres saben adónde van cuando salen mientras que dentro de los alumnos de grupos únicos lo mencionan un $21.3 \%$ equivalente a 26 adolescentes, así como el $50 \%$ equivalente a 79 alumnos de grupos regulares nos comentan que su madre lo obliga a llegar a la hora fijada a diferencia de los alumnos de grupo único donde podemos notar que hay un $16.4 \%$ equivalente a 20 adolescentes.

Por otro lado, el $27.8 \%$ equivalente a 44 alumnos de grupo regular nos dice que habla con sus madres sobre sus inquietudes sexuales, a diferencia de los alumnos de grupos únicos dentro de los cuales el $13.9 \%$ equivalente a 17 adolescentes nos mencionan lo mismo. Pudiendo observar con esto que existe una diferencia significativa entre la relación, así como una mejor comunicación y control que llevan los alumnos de grupos regulares con su madre. Con base a los resultados, puede observarse los alumnos de grupos únicos han iniciado su vida sexual, pero a pesar de que la población de grupos regulares es menor, estos, han iniciado su vida sexual a una menor edad, sin el uso de preservativos. 
Por otro lado, podemos observar que tanto los grupos de grupos únicos y regulares tienen comunicación con su padre en cuanto a su vida y sus problemas, pero hay poco control por parte de estos sobre los lugares a donde sale y la hora de llegada. Todo lo contrario, en la relación con la madre en la que notamos que hay una diferencia significativa en la relación entre alumnos de grupos únicos y regulares y su madre. Siendo notorio que hay una mejor comunicación y control entre los alumnos de grupos únicos y sus madres.

\section{Consumo de sustancias}

\section{Tabaco}

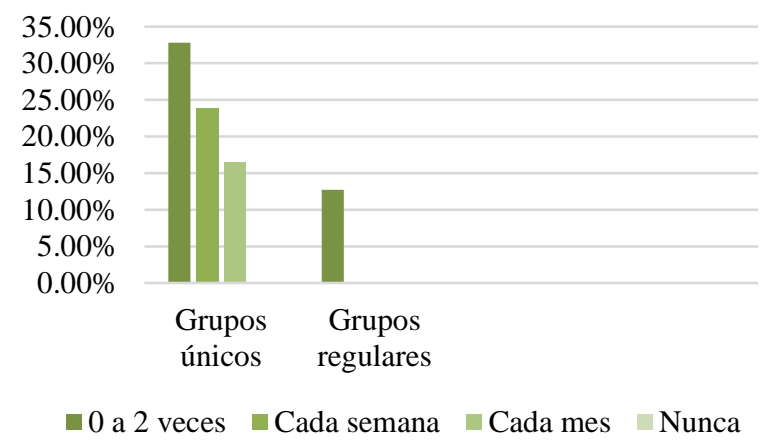

\section{Gráfico 7}

Con respecto a este apartado, las siguientes graficas muestran los reactivos más importantes para conocer la incidencia de los alumnos de grupos únicos y grupos regulares en el uso y abuso de sustancias.

\section{Consumo de sustancias a lo largo de su vida}

Esta variable mide si ha existido algún consumo de sustancias a lo largo de su vida. Podemos notar que en los grupos únicos con un $82 \%$ equivalente a 100 alumnos han consumido alguna sustancia alguna vez en su vida, mientras que el $18 \%$ nos dice que no ha consumido, mientras que en los grupos regulares con un $47.5 \%$ equivalente a 75 alumnos nos dice que, si ha consumido alguna sustancia alguna vez en su vida, mientras que el $52.5 \%$ equivalente a 83 alumnos nos dice que no ha consumido alguna sustancia. Pudiendo notar que, aunque no hay una gran diferencia en la frecuencia de consumo entre los dos grupos, hay una mayor población de alumnos de grupos únicos que han consumido alguna vez.
¿Ha consumido alguna sustancia a lo largo de su vida?

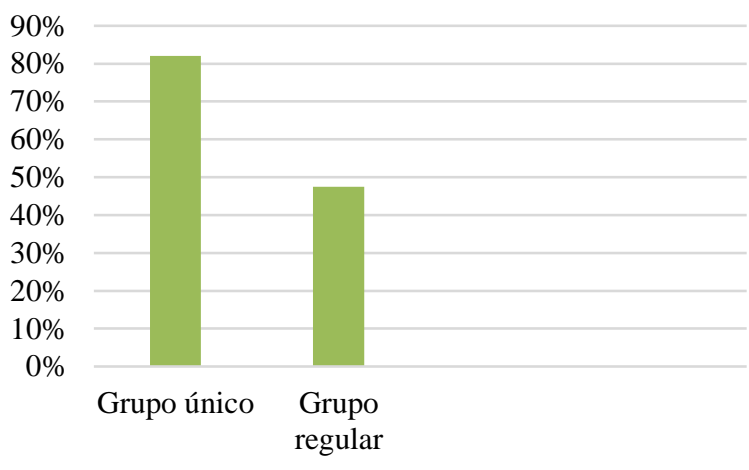

\section{Gráfico 8}

\section{Consumo de sustancia en los últimos 3 meses}

\section{Tabaco}

Esta variable mide la incidencia en los últimos 3 meses en el consumo de tabaco.

En los Gráficos podemos notar que los alumnos de grupos únicos con un 32.8\% equivalente a 40 personas han consumido de 0 a 2 veces tabaco en los últimos 3 meses, un $23.8 \%$ equivalente a 29 personas cada semana, $16.4 \%$ equivalente a 20 personas cada mes, mientras que los alumnos de grupos regulares con un $12.7 \%$ equivalente a 20 personas han consumido tabaco de 0 a 2 veces los últimos 3 meses. Indicándonos que la incidencia del consumo de tabaco es mayor en los grupos únicos que regulares.

\section{Bebidas alcohólicas}

Esta variable mide la incidencia en los últimos 3 meses en el consumo de bebidas alcohólicas.

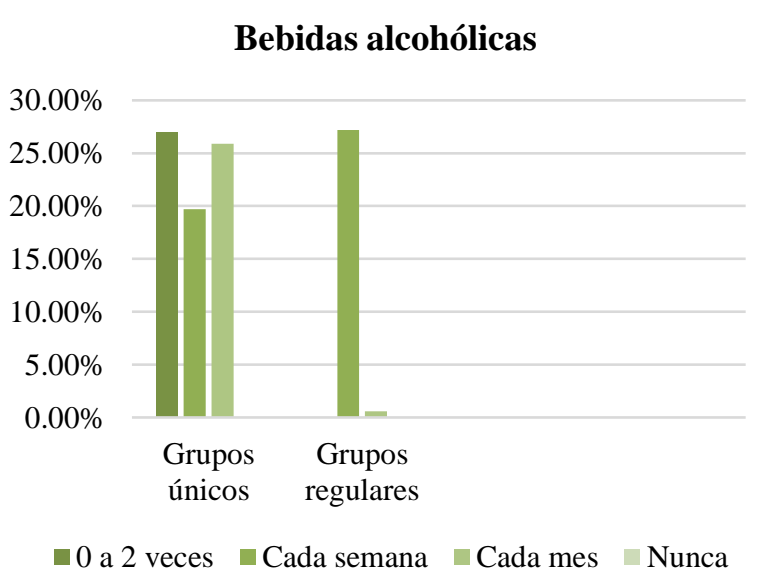

\section{Gráfico 9}


En los gráficos podemos notar que los alumnos de grupos únicos con un $27.0 \%$ equivalente a 33 personas han consumido de 0 a 2 veces bebidas alcohólicas en los últimos 3 meses, un $25.4 \%$ equivalente a 31 personas cada mes, $19.7 \%$ equivalente a 24 personas cada semana, mientras que los alumnos de grupos regulares con un $27.2 \%$ equivalente a 43 personas han consumido bebidas alcohólicas de 0 a 2 veces los últimos 3 meses y un $0.6 \%$ equivalente 1 persona ha consumido alcohol cada mes. Indicándonos que la incidencia del consumo bebidas alcohólicas es mucho mayor en los grupos únicos que regulares.

\section{Cannabis}

Esta variable mide la incidencia en los últimos 3 meses en el consumo de Cannabis.

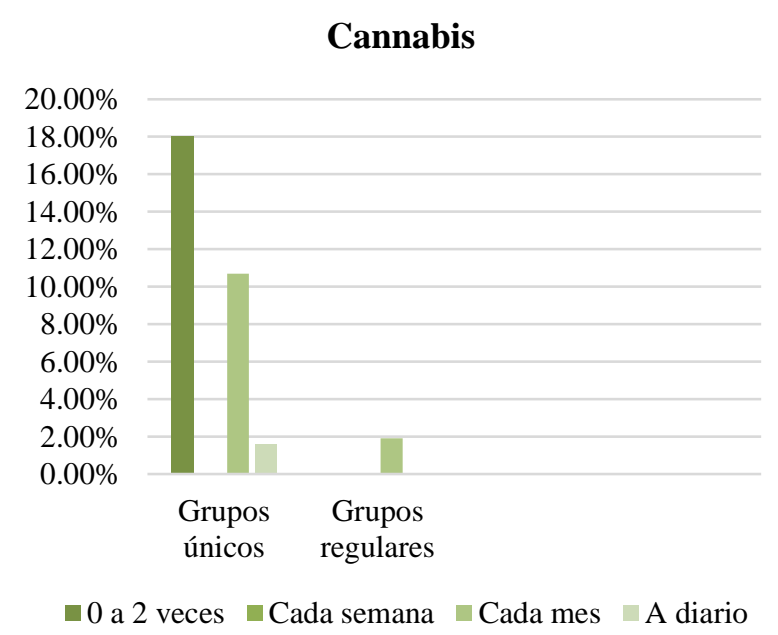

\section{Gráfico 10}

Un $18.0 \%$ equivalente a 22 personas del grupo único han consumido cannabis de 0 a 2 veces en los últimos 3 meses. Un $10.7 \%$ equivalente a 13 personas cada mes, $1.6 \%$ equivalente a 2 personas ha consumido diario. Los alumnos de grupos regulares con un $1.9 \%$ equivalente a 3 personas han consumido cannabis cada mes. Indicándonos que la incidencia del consumo de cannabis es mucho mayor en los grupos únicos que regulares.

\section{Cocaína}

Esta variable mide la incidencia en los últimos 3 meses en el consumo de cocaína.

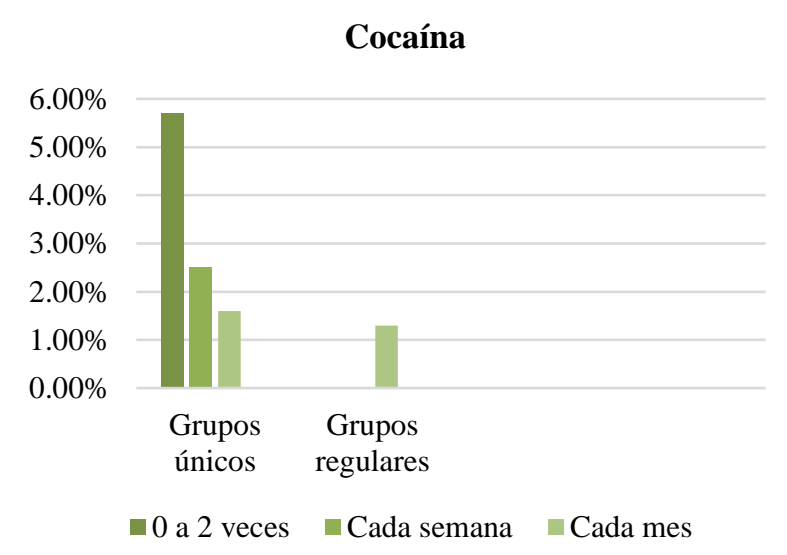

\section{Gráfico 11}

En los gráficos podemos notar que los alumnos de grupos únicos con un $5.7 \%$ equivalente a 7 personas han consumido de 0 a 2 veces cocaína en los últimos 3 meses, un $2.5 \%$ equivalente a 3 personas cada semana, $1.6 \%$ equivalente a 2 personas ha consumido cada mes, mientras que los alumnos de grupos regulares con un $1.3 \%$ equivalente a 2 personas han consumido cocaína cada mes. Indicándonos que la incidencia del consumo de cocaína es mayor en los grupos únicos que regulares.

\section{Anfetaminas}

Esta variable mide la incidencia en los últimos 3 del consumo de anfetaminas.

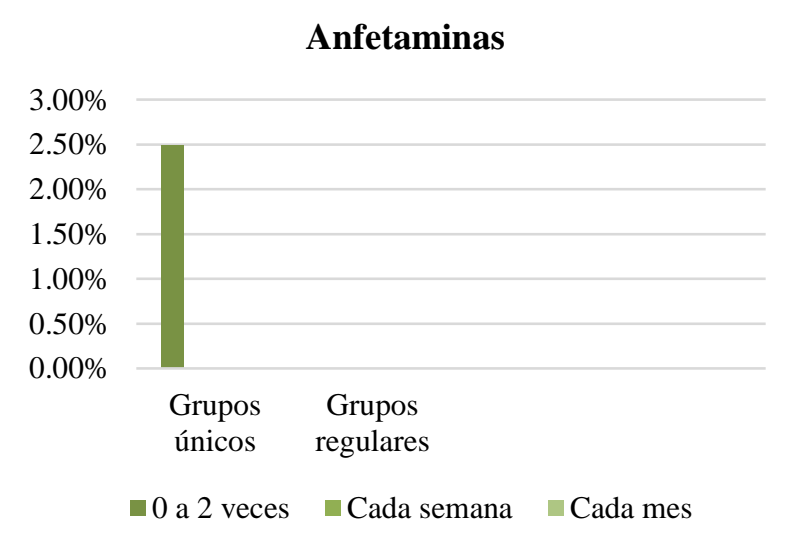

Gráfico 12

Los gráficos nos indican que los alumnos de grupos únicos con $2.5 \%$ equivalente a 3 personas han consumido anfetaminas de 0 a 2 veces, mientras que los alumnos de grupos regulares nunca han consumido anfetaminas en su vida. 
Con base a los resultados obtenidos en la prueba ASSIST podemos observar que hay una mayor población en los alumnos de grupos únicos que ha consumido alguna vez en su vida alguna sustancia, con un equivalente a 100 adolescentes. Así como que en los últimos tres meses tienen una mayor incidencia en el consumo de bebidas alcohólicas, tabaco, cannabis y cocaína qué los alumnos de grupos regular. De igual forma una minoría tanto de alumnos de grupos únicos como de regulares han consumido en los últimos 3 meses tranquilizantes y anfetaminas.

\section{Conclusiones}

Con base a los resultados obtenidos se concluye:

Referente a la sintomatología depresiva, se observan altos índices en los grupos regulares en los síntomas como "sentí que no podía quitarme la tristeza ni con la ayuda de familia ni amigos", "problemas para poner atención", "me sentí deprimido", "pensé que mi vida era un fracaso", "dormía sin descansar", "me sentía solo", los cuales no nos indican criterios suficientes para ser diagnosticados como depresión, pero si son síntomas que causan inquietud en esta población.

Con respecto a ideación suicida, se observan nuevamente altos índices en los alumnos de grupos regulares en el ítem "pensé en matarme" con un porcentaje de $16.7 \%$ equivalente a 25 alumnos que menciona tener este pensamiento de entre 5 a 7 días. Acerca del área de sexualidad, se observan altos índices en los grupos únicos con un $81 \%$ de incidencia de haber tenido la primera experiencia sexual, así como entre los alumnos de grupos regulares hay un índice significativo de personas que dicen nunca utilizar condón en sus relaciones sexuales, y los alumnos de grupos regulares la mayoría siempre utiliza condón en sus relaciones sexuales. Acerca del uso y abuso de sustancias, existe un porcentaje significativo entre los alumnos de grupos únicos que han consumido mayormente tabaco, alcohol, cannabis y con menor frecuencia cocaína y anfetaminas con una incidencia que varía desde cada semana y cada mes, siendo esto preocupante ya que a pesar de ser adolescentes tienen acceso a drogas ilegales que podrían crear una adicción significativa.
En cuanto a la relación con los padres podemos observar que tanto los grupos de grupos únicos y regulares tienen comunicación con su padre en cuanto a su vida y sus problemas, pero hay poco control por parte de estos sobre los lugares a donde sale y la hora de llegada. Todo lo contrario, en la relación con la madre en la que notamos que hay una diferencia significativa en la relación entre alumnos de grupos únicos y regulares y su madre. Siendo notorio que hay una mejor comunicación y control entre los alumnos de grupos únicos y sus madres.

En general, concluimos con que los alumnos de los grupos únicos del plantel 01 "Tuxtla Terán" inciden con mayor frecuencia en conductas de riesgo psicosocial como consumo y abuso de sustancias como tabaco, alcohol, cannabis y cocaína, así como nos percatamos que en cuanto a rasgos depresivos los alumnos de grupos regulares fueron los que obtuvieron mayor incidencia esto puede traducir a un menor rendimiento académico por parte de los alumnos de los grupos únicos, a la par de un mayor rendimiento académico de los alumnos de grupos regulares. Concluyendo que no se encuentra relación en ser alumno de grupo único con incidir en conductas de riesgo

\section{Referencias}

Aluja, A., Del Barrio, V. y García, L. F. (2005). Relation ships between adolescents memory of parental rearmg styles, social values and socialization behaviortraits. Personality and Individual Differences, 39, 903-912. Doi: 10.1016/j.paid.2005.02.028

Antolín, L., Oliva, A. y Arranz, E. (2009). Variables familiares asociadas a la conducta antisocial infantil: el papel desempeñado por el tipo de estructura familiar. Apuntes de Psicología, 27, 475-487.

Dryfoos, J. (1990). Adolescents at risk: Prevalence and prevention. New York: Oxford University Press.

Jaureguizar, J. e Ibabe, I. (2012). Conductas violentas de los adolescentes hacia las figuras de autoridad: el papel mediador de las conductas antisociales. Revista de Psicología Social, 27, 7-24. Doi: $10.1174 / 021347412798844088$.

HERNÁNDEZ-GORDILLO, José Luis, ESCALANTE-GORDILLO, Aurora Fernanda, LOPEZ-HERNÁNDEZ, Luvia Citlalli y RUÍZFLORES, Ana María. Conductas de riesgo psicosocial en alumnos del Plantel 01 (Colegio de Bachilleres de Chiapas). Revista de Didáctica Práctica. 2019 
Jessor, R. (1991). Risk behavior in adolescence: A psychological framework for understanding and action. Journal of Adolescent Health, 12, 597-605.

M. E. Lamb (Ed.). The role of the father in child development (Pp. 212-226). Nueva York: Wiley and Sons.

OMS (2019) Salud mental. Depresión. Ginebra, Suiza. Sitio web mundial. Recuperado de https://www.who.int/mental_health/managemen $\mathrm{t} /$ depression/es/

Papalia, D., (2009). Desarrollo de la infancia a la adolescencia. Undécima edición. Universidad autónoma de México.

Rodrigo, M.J., García, M., Márquez, M.L. y Triana, B. (2005). Discrepancias entre padres e hijos adolescentes en la frecuencia percibida e intensidad emocional en los conflictos familiares. Estudios de Psicología. Universidad del país Vasco: España. 26, 21-34.

Werner, E. (1989). High risk children in young adulthood: A longitudinal study from birth to 32 years. American Journal of Orthopsychiatry, 59, 59-71. Doi: j.1939-0025.1989.tb01636.x 